\title{
The Utility of Disposable Negative Pressure Wound Therapy (PICO) in Wound Healing of Latissimus Dorsi Musculocutaneous Flap Donor Sites
}

\author{
Jeoung Hyun Nam (10), Eun Soo Park 미, Seok Hwan Kim (1) \\ Department of Plastic and Reconstructive Surgery, Soonchunhyang University Bucheon Hospital, Bucheon, Korea
}

\begin{abstract}
Background: Among treatments aiming to solve surgical wound complications, negative pressure wound therapy (NPWT) is considered an innovative method. NPWT can promote wound healing, protect the wound from infection and reduce the tension on suture sites. At the same time, the large machine required in the therapy led to some inconvenience. The PICO system has recently been developed as a simple pocket-sized NPWT device. By comparing the time required for healing of the wound, incidence of wound complications, duration of hospital stays, and dressing costs, we attempted to confirm the utility of PICO for managing latissimus dorsi musculocutaneous flap donor sites. Methods: PICO was used on nine donor sites of patients who had undergone breast reconstruction using latissimus dorsi musculocutaneous flaps. PICO was applied immediately after operation and removed on the 9th day. In the control group, daily conventional dressings were administered commensurate with the condition of the wounds. We defined the wound healing time to the point when no more dressing was needed. For the costs of dressing, only costs incurred on the donor sites were included.

Results: Wound healing was proven to be faster in the PICO group $(\mathrm{P}=0.035)$ versus the control group, and no complications were observed in the PICO group. Also, the cost of PICO was lower compared to the costs incurred by the conventional dressing method (P<0.001).

Conclusion: We suggest that PICO can reduce wound complications on areas where anatomical movement could lead to tension, while also reducing dressing costs.
\end{abstract}

Keywords: Negative-pressure wound therapy; Wound healing; Surgical flaps

\section{Introduction}

The skin and soft tissues of the back, anterior chest wall and joints are inherently subject to tension, making healing of wounds in these areas difficult. Such wounds often present complications such as disruption, delayed healing and scarring. For example, reconstructive surgeons often use the latissimus dorsi (LD) muscle for breast reconstruction and frequently there are problems for wound healing of donor sites. Numerous trials and studies to facilitate healing of wounds in such areas have either been carried out or are in progress.

In the American College of Surgeons National Surgical Quality Improvement Program Participant User File (ACS-NSQIP PUF), there were 19,361 cases that underwent immediate breast reconstruction using the LD flap from 2005 to 2014 because of breast malignancy [1]. Meanwhile, in the study of Yezhelyev et al. [2], 277 patients underwent breast reconstruction using LD musculocutaneous flaps. Of the 277, donor site complications occurred in 62 patients (22.3\%), among which three patients (1.1\%) suffered from skin necrosis. Skin necrosis may result in scars and require additional surgical procedures. Extrapolating from the statistics in Yezhelyev et al. [2], we
Original Article

Received: December 2, 2019

Revised: February 4, 2020

Accepted: February 5, 2020

\section{Corresponding author:}

Eun Soo Park, M.D., Ph.D.

Department of Plastic and Reconstructive Surgery, Soonchunhyang University Bucheon Hospital, 170 Jomaru-ro, Wonmi-gu, Bucheon 14584 , Korea

Tel: +82-32-621-5319

Fax: +82-32-621-5016

E-mail: peunsoo@schmc.ac.kr

This article was presented as a poster at the Aesthetic Plastic Surgery on April 6-7, 2019, in Seoul, Korea.

This is an Open Access article distributed under the terms of the Creative Commons Attribution Non-Commercial License (https://creativecommons.org/licenses/by-nc/4.0/) which permits unrestricted non-commercial use, distribution, and reproduction in any medium, provided the original work is properly cited.

C) 2020 Korean Wound Management Society 
can infer that a significant number of patients suffer from problems in wound healing on their donor sites after breast reconstruction using LD musculocutaneous flaps.

Negative pressure wound therapy (NPWT) is one of the proven treatments for wound healing of the suture site [3,4]. NPWT promotes wound healing in the closed suture site by removing fluid from the incisional interspaces, acting as a splint against distracting forces, protecting from environmental contamination, and promoting upregulation in the expression of vascular endothelial growth factor [5]. However, for most NPWT systems there exist drawbacks such as the noise of the machinery and restrictions in movement due to the significant size of the machine. In addition, the considerable price of the machine makes it difficult to discharge patients to continue using the machine at home. Thus, the PICO system has been developed, providing a simple, single-use device that fits in the hand, which can also be used on incisional wounds (Fig. 1). It delivers a continuous negative pressure of $80 \mathrm{mmHg}$ on sealed wounds and can absorb up to $300 \mathrm{~mL}$ of exudate during 1 week of therapy. The top film layer acts as a physical barrier and allows the discharge to evaporate [6]. The compact device is much more convenient and affordable for patients.

Donor sites of LD musculocutaneous flaps sometimes suffer from skin necrosis and suture complications. Though conventional NPWT can be conducive in the healing of donor sites, the unwieldy size of the machine and the noise it emanates make the use of the system inconvenient. Therefore, in this study, we aimed to confirm the utility of the smaller and more convenient PICO in managing donor sites for breast reconstruction that used LD musculocutaneous flaps.

\section{Methods}

\section{Patients' characteristics}

The retrospective chart review was done on patients who had
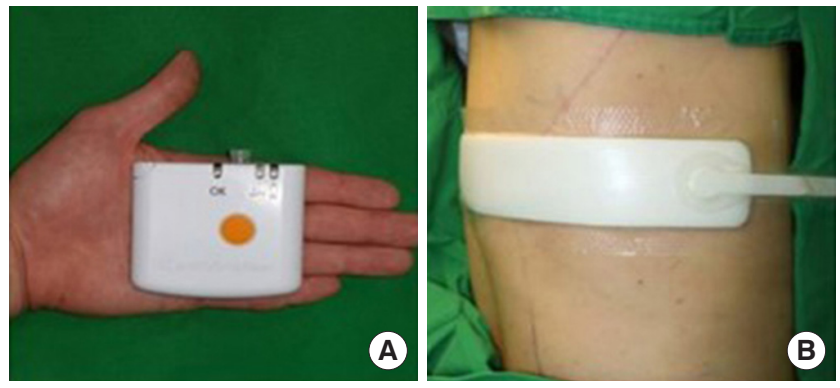

Fig. 1. Disposable negative pressure wound therapy device (PICO). (A) The handful size of device. (B) The applied state of device. operations from March 2018 to March 2019. Patients with factors that may influence wound healing [7] (i.e., diabetes, keloid, steroid medication, smoking, alcoholism, history of radiation, immunocompromised conditions, and age $>60$ years) were excluded from the study. We obtained written informed consent for the use of photographs before the operations took place. We conducted our research in compliance with the principles of the Declaration of Helsinki.

\section{Surgical methods}

For smooth primary closure, the entire skin paddle was designed with a width to length ratio of 1:3 to 1:4 (Fig. 2). The skin paddles were designed similarly at the inframammary line level for all patients. The fascia was closed using polydioxanone (PDS) No. 2 thread and the subcutaneous layer was sutured with PDS No. 4. Finally, the skin was sutured with nylon No. 4 to keep the suture sites of all the patients as similar as possible.

\section{Dressing methods}

In the experimental group, PICO was applied immediately after the operation, changed after the 4th day, and removed on the 9th day. In the control group, the wound was covered with Medifoam (Ildong Pharmaceutical Co., Ltd., \& Biopol Co., Ltd., Seoul, Korea; a hydrocellular semi-permeable dressing material) after applying Fusimed Ointment (Green Cross Corp., Yongin, Korea; a fusidic acid) once a day. When there were problems such as infections or necrotic changes, appropriate dressing was provided using Vaseline Yunna Gauze (Q\&Q Medic Co., Ltd., Seoul, Korea), Betadine solution (Korea Pharma, Seoul, Korea; povidone-iodine, 10\%), etc. Half the stitches were taken out on the 10th day after the operation, and

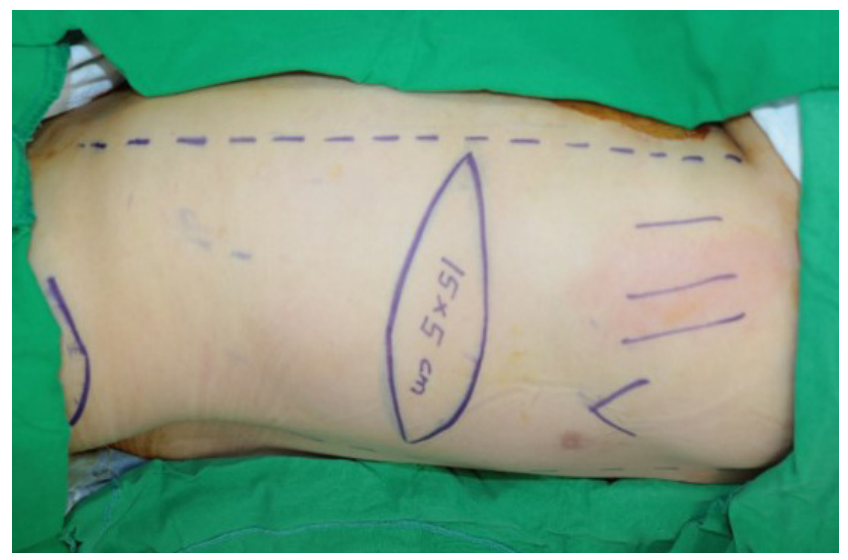

Fig. 2. Preoperative skin paddle design. 
all remaining stitches were removed on postoperative day 11 . If coaptation was not observed, removal of the stitches was postponed. Abnormal wound states such as suture site disruption and eschar formation were considered wound complications, and we defined the wound healing time as to the point when no more dressing was needed. Only costs incurred on the donor sites were included when assessing the cost of dressing.

\section{Scar evaluation}

After total stitch removal, scar evaluation of the suture site was performed using the Stony Brook Scar Evaluation Scale [8]. Width, height, color, suture marks, and overall appearance were evaluated with 1 point given for each favorable aspect for a maximum total of 5 points. This scale was proposed in 2007 by Singer et al. [8] to measure the short-term cosmetic outcome of wounds 5 to 10 days after removing the sutures.

\section{Analysis}

Statistical analyses were performed using IBM SPSS Statistic version 19.0 (IBM Corp., Armonk, NY, USA). Because the sample size was small, groups were compared using the Mann-Whitney U-test, and the occurrence of complications were calculated with Fisher exact test.
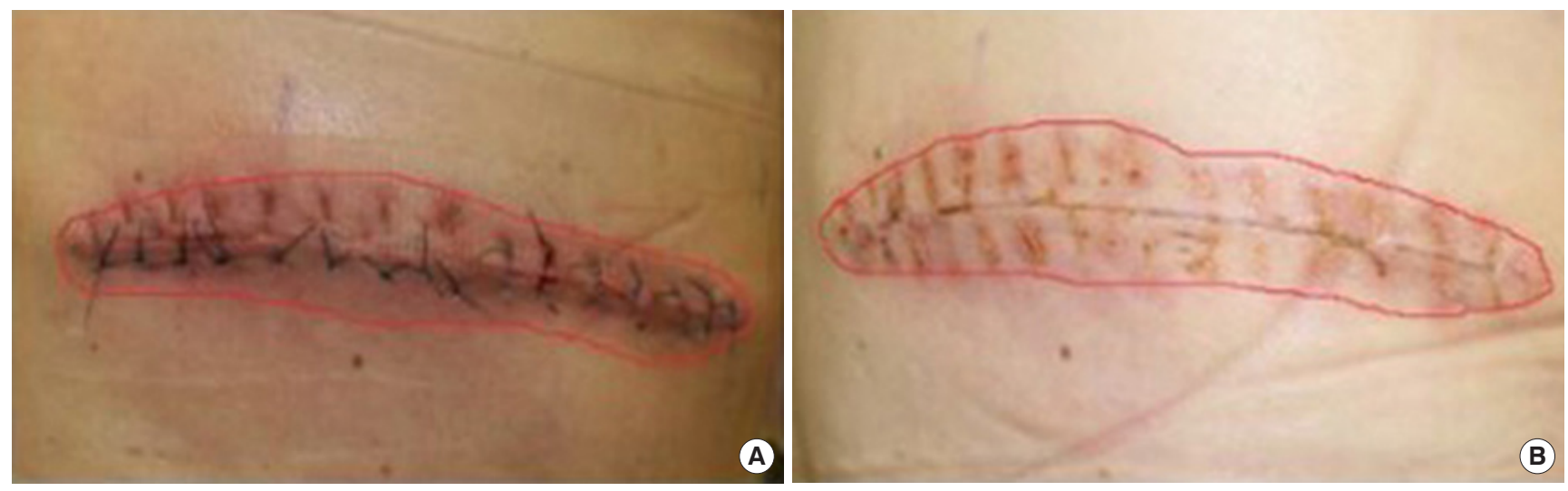

Fig. 3. The wound of dressing group. (A) Postoperation 4th day. (B) Postoperation 12th day.
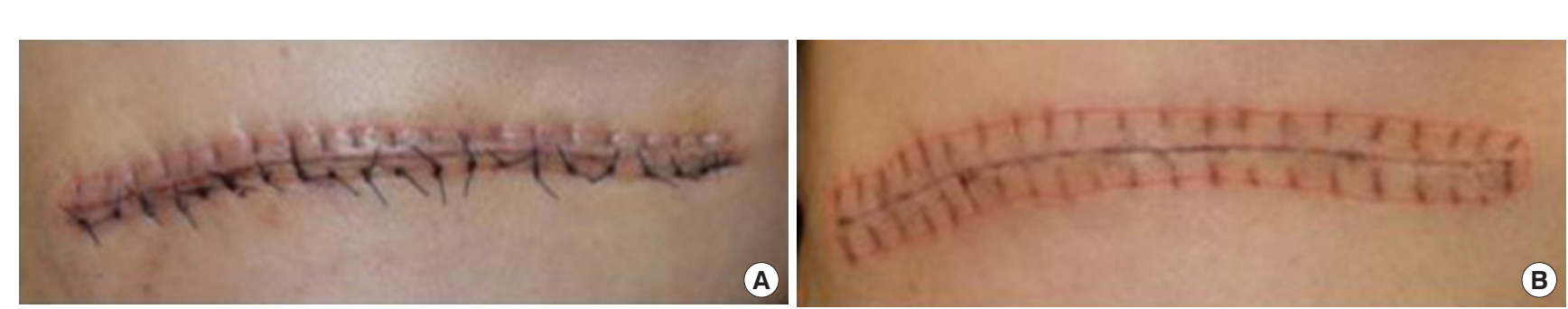

Fig. 4. The wound of PICO group. (A) Postoperation 4th day. (B) Postoperation 12th day.

\section{Results}

From March 2018 to March 2019, 19 patients received immediate breast reconstruction using the LD musculocutaneous flap after mastectomy. PICO was applied to nine patients and conventional dressing was provided for 10 patients. No wound complications were observed in the PICO group (0\%) (Figs. 3, 4 ), while there were three cases of wound complications in the conventional dressing group (30\%). In two of the three cases, we dressed the wounds with Betadine solution to respond to infection, and in the other case, we performed moisture dressing using Vaseline Yunna Gauze after debridement to treat skin necrosis. The P-value was 0.211 and there was no statistical difference in wound complications (Table 1). The cost of dressing in the PICO group was Korean Won (KRW) 120,191 and in the conventional dressing group, the cost was KRW strating statistical significance with a P-value $<0.001$. As for wound healing time, the PICO group took 11.44 days while the conventional dressing group required 13.20 days, resulting in a statistically significant P-value of 0.035 . The scar evaluation scores were 3.89 in the PICO group and 0.80 in the conventional dressing group, with a P-value lower than 0.001 , 250,465 (costing KRW 20,711 per dressing), thereby demon- 
Table 1. Wound complication rate

\begin{tabular}{lccc}
\hline & PICO group & $\begin{array}{c}\text { Conventional dressing } \\
\text { group }\end{array}$ & P-value \\
\hline Without complication & $9(100)$ & $7(70)$ & 0.211 \\
With complication & 0 & $3(30)$ & \\
\hline
\end{tabular}

Values are presented as number (\%).

which was still statistically significant (Table 2 ). In addition, the fact that daily dressing was not required helped save costs for the PICO group. Complications such as skin irritation, dermatitis and abrasions that usually arise while applying Mefix (SCA Molnlycke Ltd.) and skin tape for Medifoam were not observed. Apparently, the inconvenience caused to the patients was reduced for the PICO group. Although the exact mechanism was not identified, decrease in the rate of seroma incidence after hemovac removal was observed. Even if the width of PICO was shorter than the $10.0 \mathrm{~cm}$ length of the dissection, it is believed to have had a compression effect on the site. It also acts as a splint and limits the patient's movement, further contributing to the healing.

\section{Discussion}

Although medical technology has made great strides in the last few decades, traditional methods of primary incision closure, as well as dressing cover over surgical incisions, have not advanced to a great extent. Many methods, including debriding agents, topical antimicrobial dressings, and nonadherent dressings have been used with an aim to stimulate the proliferative phase of wound healing. However, their use demonstrated limited efficacy and resulted in minimal improvement. But with the introduction of NPWT therapy in the 1990s, which is delivered by a portable and practical device, the landscape of wound management has been drastically altered [9]. However, there remain limitations to routine use because of problems of portability and price. Attempts to solve this problem have been made, leading to invention of the lightweight and cheap incisional NPWT system. The device acts as a splint and offloads tension on the skin incisions, thereby reducing resistance and tension on the entire wound. However, several problems with PICO were discovered during the course of the study. First, detection of complications may be delayed as the wound is checked only on the 4th and 9th days. Also, since it is a disposable machine, the durability of the device is often problematic; it may break upon dropping.
Table 2. Dressing cost, wound healing time, and scar evaluation score

\begin{tabular}{lccr}
\hline Variable & PIC0 group & $\begin{array}{c}\text { Conventional dressing } \\
\text { group }\end{array}$ & P-value \\
\hline Dressing cost (KRW) & 102,191 & 250,465 & $<0.001$ \\
Wound healing time (day) & 11.44 & 13.20 & 0.035 \\
Scar evaluation score & 3.89 & 0.80 & $<0.001$ \\
\hline
\end{tabular}

Values are presented as mean.

KRW, Korean Won.

There were several limitations to this study. While it is common to replace the conventional dressings once every 2-3 days after a few days, the dressing would detach easily because it was placed where there was frequent movement. Daily dressing was also provided to the conventional dressing group because most inpatients demand their wounds to be dressed and examined every day, increasing the costs. In addition, since all the patients' sutures were not done by the same surgeon, it was not possible to create uniformly ideal conditions across all the suture sites. Also, because we did not factor in the possible hemovac and seroma complications when we were designing the study, we were not able to statistically analyze their effect. Such analysis and also examination of long term scarring should be included in further studies.

In conclusion, PICO is believed to be helpful in stabilizing and healing wounds $(\mathrm{P}=0.035)$ by reducing the tension on the wounds and at the same time acting as a splint in the movable area. In addition, it costs less $(\mathrm{P}<0.001)$ and requires less manpower compared to conventional dressings. Because patients also feel less discomfort, it is strongly recommended as a replacement for dressing on the back.

\section{Conflict of interest}

No potential conflicts of interest relevant to this article are reported.

\section{ORCID iDs}

Jeoung Hyun Nam https://orcid.org/0000-0002-2151-5869

Eun Soo Park https://orcid.org/0000-0003-2966-9122

Seok Hwan Kim https://orcid.org/0000-0003-1859-5703

\section{References}

1. Ilonzo N, Tsang A, Tsantes S, et al. Breast reconstruction 
after mastectomy: a ten-year analysis of trends and immediate postoperative outcomes. Breast 2017;32:7-12.

2. Yezhelyev M, Duggal CS, Carlson GW, et al. Complications of latissimus dorsi flap breast reconstruction in overweight and obese patients. Ann Plast Surg 2013;70:557-62.

3. Huang C, Leavitt T, Bayer LR, et al. Effect of negative pressure wound therapy on wound healing. Curr Probl Surg 2014;51:301-31.

4. Scalise A, Calamita R, Tartaglione C, et al. Improving wound healing and preventing surgical site complications of closed surgical incisions: a possible role of incisional negative pressure wound therapy. A systematic review of the literature. Int Wound J 2016;13:1260-81.

5. Shah A, Sumpio BJ, Tsay C, et al. Incisional negative pressure wound therapy augments perfusion and improves wound healing in a Swine Model Pilot Study. Ann Plast Surg 2019;82(4S Suppl 3):S222-7.

6. Payne C, Edwards D. Application of the single use negative pressure wound therapy device (PICO) on a heterogeneous group of surgical and traumatic wounds. Eplasty 2014;14: e20.

7. Guo S, Dipietro LA. Factors affecting wound healing. J Dent Res 2010;89:219-29.

8. Singer AJ, Arora B, Dagum A, et al. Development and validation of a novel scar evaluation scale. Plast Reconstr Surg 2007;120:1892-7.

9. Conde-Green A, Chung TL, Holton LH 3rd, et al. Incisional negative-pressure wound therapy versus conventional dressings following abdominal wall reconstruction: a comparative study. Ann Plast Surg 2013;71:394-7. 